\title{
Age-Related Prevalence of Periodontoid Calcification and Its Associations with Acute Cervical Pain
}

\author{
Takashi Kobayashi ${ }^{1}$, Naohisa Miyakoshi ${ }^{2}$, Norikazu Konno ${ }^{1}$, \\ Yoshinori Ishikawa ${ }^{2}$, Hideaki Noguchi $^{2}$, Yoichi Shimada ${ }^{2}$ \\ ${ }^{I}$ Department of Orthopedic Surgery, Akita Kousei Medical Center, Akita, Japan \\ ${ }^{2}$ Department of Orthopedic Surgery, Akita University Graduate School of Medicine, Akita, Japan
}

\begin{abstract}
Study Design: Prospective study.
Purpose: To assess the prevalence of periodontoid calcification and its associations with acute cervical pain.

Overview of Literature: Calcium pyrophosphate dihydrate (CPPD) deposition disease is a common rheumatological disorder that occurs especially in elderly patients. Although CPPD crystals induce acute arthritis, these crystals are not usually symptomatic. Calcification surrounding the odontoid process (periodontoid calcification) has been reported to induce inflammation, resulting in acute neck pain. This disease is called crowned dens syndrome. Whether calcification induces inflammation or whether the crystals are symptomatic remains unclear.

Methods: The prevalence of periodontoid calcification at the atlas transverse ligament was examined by computed tomography of the upper cervical spine in patients suspected of brain disease but no cervical pain (control group, $n=296$ ), patients with pseudogout of the peripheral joints but no cervical pain (arthritis group, $\mathrm{n}=41$ ), and patients with acute neck pain (neck pain group, $\mathrm{n}=22$ ). Next, the correlation between the prevalence of periodontoid calcification and symptoms was analyzed.

Results: In the control group, 40 patients (13.5\%) showed periodontoid calcification with no significant difference in the prevalence with gender. The prevalence of calcification increased significantly with age $(p=0.002)$. In the arthritis group, 26 patients $(63.4 \%)$ reported periodontoid calcification. In the neck pain group, 14 patients (63.6\%) reported periodontoid calcification. Multiple logistic regression analysis by age and group revealed that higher age, inclusion in the arthritis group, and inclusion in the neck pain group significantly affected the prevalence of calcification.

Conclusions: Our results cumulatively suggest that periodontoid calcification is an aging-related reaction and that calcification per se does not always cause neck pain. Periodontoid calcification was observed more frequently in patients with pseudogout of the peripheral joints and in those with acute neck pain than in asymptomatic control patients.
\end{abstract}

Keywords: Arthritis; Calcification; Calcium pyrophosphate; Chondrocalcinosis; Neck pain

Received Feb 1, 2018; Revised Mar 23, 2018; Accepted Apr 17, 2018

Corresponding author: Takashi Kobayashi

Department of Orthopedic Surgery, Akita Kousei Medical Center, 1-1-1 Iijima-Nishifukuro, Akita 011-0948, Japan

Tel: +81-18-880-3000, Fax: +81-18-880-3040, E-mail: takakoba825@hotmail.com 


\section{Introduction}

Calcium pyrophosphate dihydrate (CPPD) deposition disease, a common rheumatologic disorder, is more frequently observed in elderly patients. Although CPPD crystals induce acute arthritis, they are not usually symptomatic in nature. Calcification surrounding the odontoid process (periodontoid calcification) has been reported to induce inflammation, which results in acute neck pain. This disease is called crowned dens syndrome [1,2]. The first such report described four patients with acute neck pain caused by periodontoid calcification [1], whereas a second series described 40 patients with severe neck pain with periodontoid calcification on computed tomography (CT) [2]. In contrast, periodontoid calcification in patients without neck pain was more frequently observed in patients diagnosed with CPPD deposition disease of the peripheral joints than in control patients [3]. Therefore, whether calcification induces inflammation or whether the crystals are symptomatic remains unclear.

This study hypothesized that periodontoid calcification does not cause acute neck pain. Accordingly, we evaluated the prevalence of periodontoid calcification in both symptomatic and asymptomatic populations and analyzed the correlation between calcification and symptoms.

\section{Materials and Methods}

All procedures involving human participants were in accordance with the ethical standards of the institutional and/or national research committee and with the 1964 Helsinki declaration and its later amendments or comparable ethical standards. Informed consent was obtained from all individual participants included in the study.

Patients were prospectively enrolled in this study during a 12 -month period. CT scans of the cervical spine were performed in three groups of patients. The control group comprised of 296 patients who underwent CT from the cervical spine to the whole brain for evaluation of suspected brain disease. These patients were consecutively admitted to Koto General Hospital for nausea, headache, dizziness, or head injury and were suspected to have brain disease, but they had no neck pain. This group represented individuals with no cervical pain in the general population. The arthritis group comprised of 41 patients with pseudogout of the peripheral joints. These patients were consecutively admitted to our hospital for peripheral
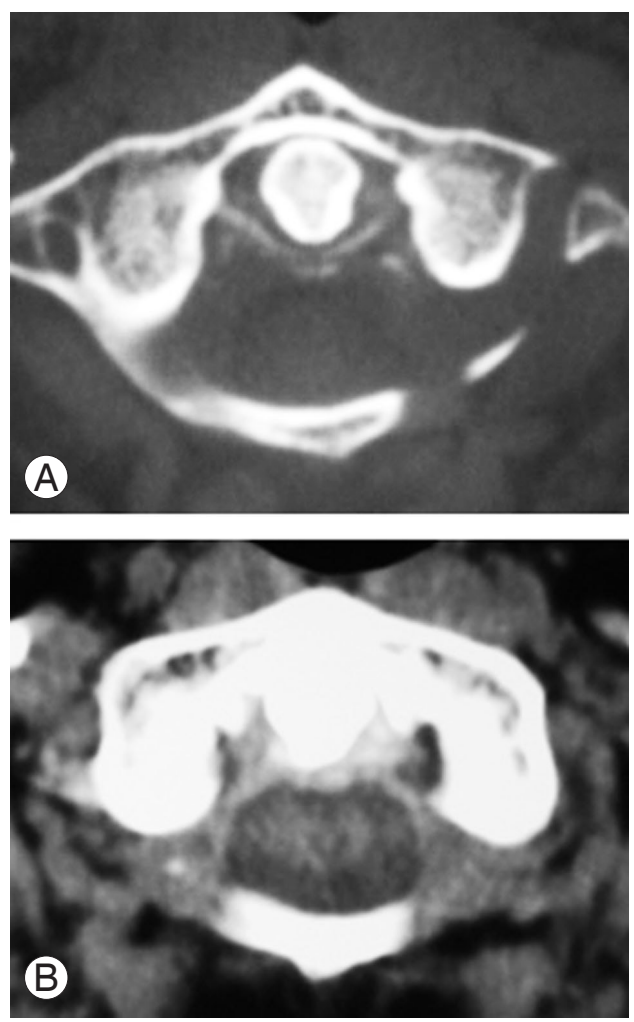

Fig. 1. Computed tomography scans of the atlas. (A) Linear type calcification. (B) Dot type calcification. The presence of either type of calcification was defined as positive for periodontoid calcification.

joint pain and diagnosed with pseudogout of the joints. All patients in this group showed no cervical pain, but after providing informed consent, they underwent $\mathrm{CT}$ of the upper cervical spine to determine whether calcification was present. The neck pain group comprised of 22 patients with acute neck pain. These patients were consecutively admitted to our hospital for treatment of neck pain and met the following criteria for CT examination of the upper cervical spine: (1) Visual Analog Scale score $>50$ $\mathrm{mm}$, (2) serum C-reactive protein level $>0.5 \mathrm{mg} / \mathrm{dL}$, and (3) tenderness in the muscles of the upper cervical spine [4].

To investigate the prevalence of periodontoid calcification in each group, non-contrast CT scans of the upper cervical spine were assessed by three orthopedic surgeons in order to determine whether calcifications were present around the odontoid process (Fig. 1). Patients in the control group were then assorted by sex and age to evaluate the age- and sex-related prevalence of calcification. Accordingly, five age groups were compared: $<50,50-59$, $60-69,70-79$, and $\geq 80$ years. 


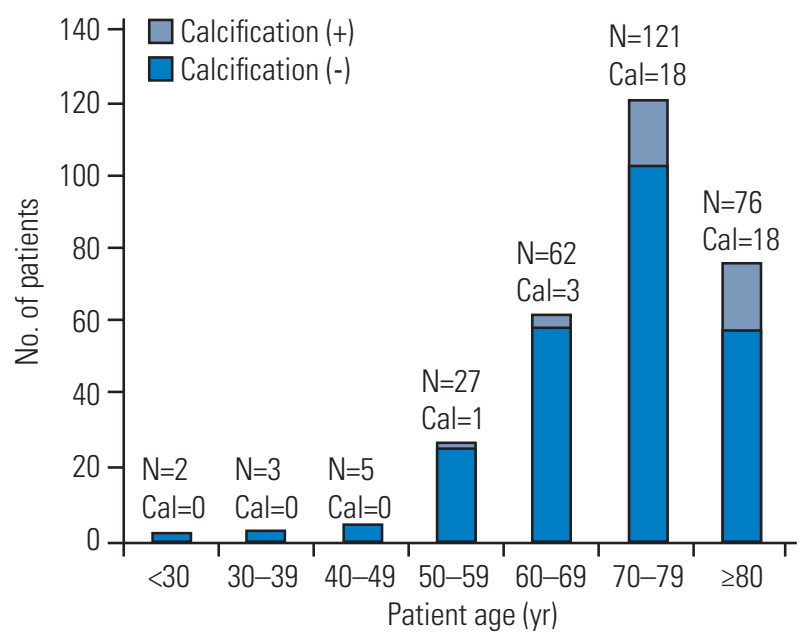

Fig. 2. Prevalence of periodontoid calcification by patient age. The prevalence of periodontoid calcification increased significantly with age (Fisher's exact test, $p=0.002$ ). Cal, calcification (t).

All $p$-values were two-sided, with $p<0.05$ considered as statistically significant. Frequency analysis was performed with Fisher's exact test. Pairwise comparisons were determined using Fisher's exact test with Holm's adjusted $p$-values. Logistic regression analysis was used to compare the prevalence of calcification among the three groups. All statistical analyses were performed with EZR ver. 1.36 (Saitama Medical Center, Jichi Medical University, Saitama, Japan), a graphical user interface for $\mathrm{R}$ (The $\mathrm{R}$ Foundation for Statistical Computing, Vienna, Austria).

\section{Results}

The control group comprised of 147 men and 149 women with the mean age of 72 years (range, 18-96 years). Of these 296 patients, 40 (13.5\%) showed periodontoid calcification, including $18(12.2 \%)$ of 147 men and 22 (14.8\%) of 149 women. The prevalence of periodontoid calcification did not differ significantly between men and women ( $p=0.61$, Fisher's exact test). None of the $10 \mathrm{pa}$ tients aged $<50$ years showed periodontoid calcification. Of the remaining patients, one $(0.03 \%)$ of the 27 patients aged $50-59$ years, three $(0.05 \%)$ of the 62 patients aged 60-69 years, $18(14.90 \%)$ of the 121 patients aged 70-79 years, and $18(23.70 \%)$ of the 76 patients aged $\geq 80$ years were positive for calcification (Fig. 2), with the prevalence of calcification increasing significantly with age (Fisher's exact test, $p=0.002$ ) (Fig. 3). Table 1 shows pairwise comparisons using Fisher's exact test with Holm's adjusted $p$ values.

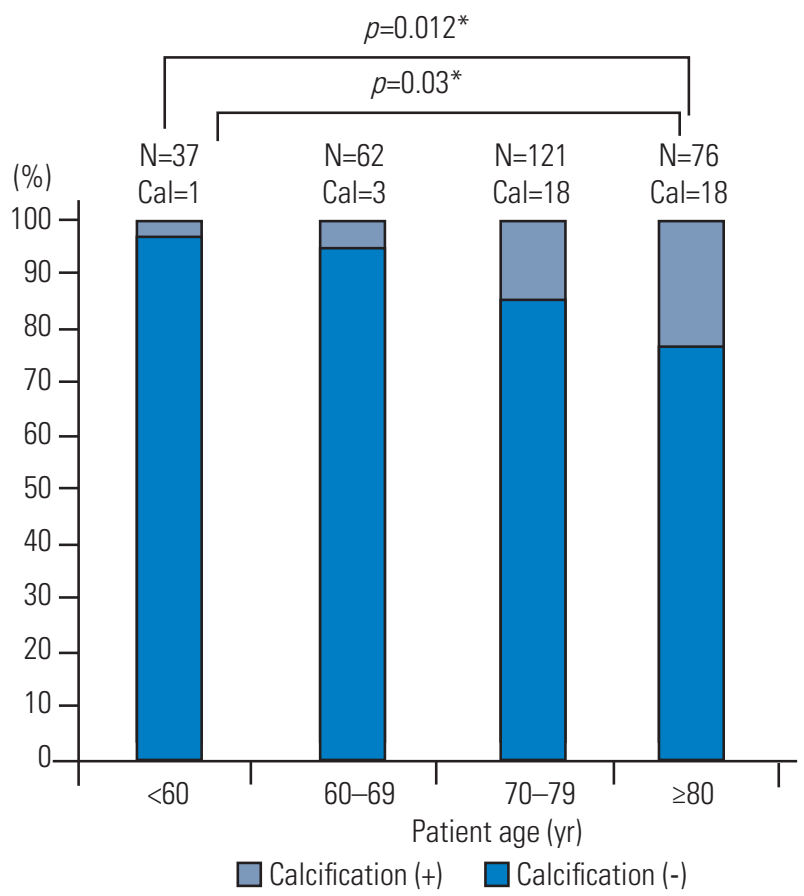

Fig. 3. Comparison of calcification prevalence with age groups. The prevalence of periodontoid calcification was significantly different among age groups (Fisher's exact test with Holm's adjusted $p$-values, $p=0.002$ ). ${ }^{*} p<0.05$.

Table 1. Pairwise comparison of the prevalence of calcification by age using Fisher's exact test with Holm's adjusted $p$-values

\begin{tabular}{llcc} 
Age (yr) & $<59$ & $60-69$ & $70-79$ \\
$60-69$ & 1.00 & - & - \\
$70-79$ & 0.19 & 0.19 & - \\
$\geq 80$ & $0.03^{*}$ & $0.012^{*}$ & 0.27 \\
\hline$p<0.05$. & & &
\end{tabular}

The arthritis group comprised of 18 men and 23 women with a mean age of 82 years (range, $59-96$ years). Of these 41 patients, 26 patients (63.4\%) showed periodontoid calcification. The affected peripheral joint was the joint of the knee in 18 patients, of the wrist in 10, of the ankle in five, of the elbow in four, of the interphalangeal joint of the thumb in two, of the carpo-metatarsal joint in one, and of the hip in one. The presence of CPPD crystals in the joint fluid was proven in 35 patients, and the other patients were diagnosed with pseudogout based on their clinical findings.

The neck pain group comprised of six men and 16 women of mean age 75.8 years (range, $52-89$ years). Of these 22 patients, 14 (63.6\%) showed periodontoid calcification. On an average, the patients first visited the hospital 2 days (range, $0-7$ days) after the onset of pain. The mean 
Table 2. Pairwise comparisons of the prevalence of calcification by group using Fisher's exact test with Holm's adjusted $p$-values

\begin{tabular}{lcccc} 
Variable & No. of cases & Age $(y r)$ & Sex (male:female) & Calcification \\
Control group & 296 & $72.0 \pm 11.1$ & $147: 149$ & $40(13.5)$ \\
Arthritis group & 41 & $82.0 \pm 7.7$ & $18: 23$ & $26(63.4)^{* *}$ \\
\hline Neck pain group & 22 & $75.8 \pm 9.4$ & $6: 16$ & $14(63.6)^{* *}$ \\
\hline
\end{tabular}

Values are presented as mean \pm standard deviation, number, or number (\%). $p<0.001$.

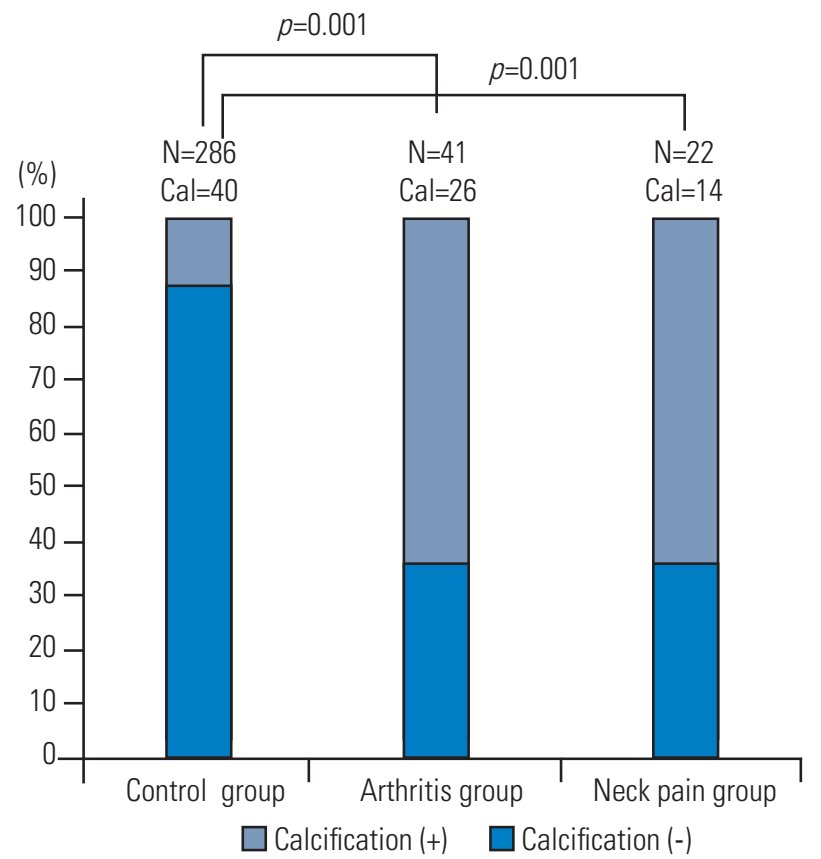

Fig. 4. Prevalence of calcification among control, arthritis, and neck pain groups. The prevalence of calcification was significantly different among the groups (Fisher's exact test with Holm's adjusted $p$-values, $p<0.001)$.

C-reactive protein level was $4.8 \mathrm{mg} / \mathrm{dL}$ (range, $0.5-14.2$ $\mathrm{mg} / \mathrm{dL}$ ). The mean visual analog scale score was $78 \mathrm{~mm}$ (range, 50-100 $\mathrm{mm}$ ).

Table 2 depicts pairwise comparisons of the prevalence of calcification around the dens by group using Fisher's exact test with Holm's adjusted $p$-values. The prevalence of calcification was significantly different among the groups $(p<0.001)$ (Fig. 4).

Multiple logistic regression analysis by age and group revealed that higher age, inclusion in the arthritis group, and inclusion in the neck pain group significantly affected the prevalence of calcification (Table 3 ).

\section{Discussion}

This study analyzed the age-related prevalence of asymp-
Table 3. Multivariate logistic regression analysis for the prevalence of calcification around the dens

\begin{tabular}{lcc} 
Variable & $\begin{array}{c}\text { Odds ratio } \\
(95 \% \text { confidence interval) }\end{array}$ & $p$-value \\
\hline Age & $0.92(0.89-0.96)$ & $<0.001$ \\
Sex & $0.75(0.42-1.36)$ & 0.34 \\
\hline Arthritis group & $0.15(0.07-0.33)$ & $<0.001$ \\
Neck pain group & $0.10(0.04-0.26)$ & $<0.001$ \\
\hline
\end{tabular}

tomatic periodontoid calcification in 296 patients without neck pain; the prevalence was compared with that in patients with pseudogout of the peripheral joints and patients with acute neck pain. Asymptomatic periodontoid calcification was identified in $13.5 \%$ of the general population who incidentally underwent upper cervical CT examination combined with brain CT; the results showed increasing prevalence with age. Our results are in good agreement with those of a previous study, which reported the presence of periodontoid calcification in four (11\%) of 35 patients with a mean age of 72 years [3].

In this study, the prevalence of periodontoid calcification in patients with pseudogout of the peripheral joints $(63.4 \%)$ or acute neck pain $(63.6 \%)$ was higher than that in the general population without neck pain (13.5\%). These results indicate that pseudogout of the peripheral joints is strongly associated with the presence of periodontoid calcification. These findings are consistent with those of several previously published studies. One study revealed a higher prevalence of calcification of the atlas transverse ligament, as assessed by axial CT in patients with articular chondrocalcinosis than in those without it (14 of 21 [66\%] versus 0 of 21 [0\%] patients, respectively) [5]. Another study showed that the prevalence was higher in patients with articular chondrocalcinosis than in those without it (24 of 35 [69\%] versus four of 35 [11\%] patients, respectively) [3].

This study also found that the prevalence of periodon- 
toid calcification increased with age in a general population. This finding is consistent with the trend in peripheral joint calcification. The crude prevalence of chondrocalcinosis in the knee reportedly ranges from $7.0 \%$ to $8.1 \%$ and shows a strong association with age [6,7]. Finckh et al. [3] reported that the prevalence of periodontoid calcification differed significantly with age. Although they found that the prevalence was higher with older age, the sample size was only 35 patients.

Acute neck pain is also associated with periodontoid calcification. Bouvet et al. [1] first reported a case of acute neck pain in association with periodontoid calcification; this condition is called as "crowned dens syndrome." This association of periodontoid calcification and severe acute neck pain was reconfirmed by Goto et al. [2] in a detailed review of the clinical records of 40 patients. Several studies also reported positive associations between acute neck pain and periodontoid calcification or crowned dens syndrome, as per CT diagnosis [1,2,8-12]. However, calcification is not always symptomatic. We may encounter asymptomatic calcification of the articular joints after $\mathrm{X}$-ray examination. In contrast, some patients develop recurrent episodes of arthritis without roentgenographic calcification [13]. Considering this background, pseudogout attacks should be diagnosed with the identification of calcium phosphate crystals in the joint fluid, but not with roentgenographic calcification [13,14]. Kobayashi et al. [4] previously reported that crystal-induced arthritis of the atlantoaxial joint is the cause of acute neck pain in elderly patients.

The two main strengths of this study include (1) inclusion of a larger number of asymptomatic control patients as compared to that in previous studies and (2) clear evidence that the prevalence of periodontoid calcification increases with age. However, this study has two main limitations: (1) the numbers of patients in the arthritis group and neck pain group were relatively small and (2) the control group comprised of patients with suspected brain disease, and therefore, they were not real 'controls.' Hence, future studies with proper disease-free controls are anticipated. Thus, investigation of the calcification morphology and its impact on neck pain can be pursued in the future.

\section{Conclusions}

This study demonstrated that the prevalence of periodontoid calcification significantly increases with age and that periodontoid calcification per se does not cause neck pain. Periodontoid calcification was noted more frequently in patients with pseudogout of the peripheral joints and in patients with acute neck pain than in asymptomatic controls.

\section{Conflict of Interest}

No potential conflict of interest relevant to this article was reported.

\section{Acknowledgments}

We thank Angela Morben, DVM, ELS, from Edanz Group (www.edanzediting.com/ac), for editing a draft of this manuscript.

\section{Author Contributions}

TK was the major contributors in writing the manuscript. TK conceived the idea of the study, and TK and NM designed the study. Data acquisition was performed by NK, YI, and HN, and YS supervised whole work. All authors read and approved the final manuscript.

\section{References}

1. Bouvet JP, le Parc JM, Michalski B, Benlahrache C, Auquier L. Acute neck pain due to calcifications surrounding the odontoid process: the crowned dens syndrome. Arthritis Rheum 1985;28:1417-20.

2. Goto S, Umehara J, Aizawa T, Kokubun S. Crowned dens syndrome. J Bone Joint Surg Am 2007;89:27326.

3. Finckh A, Van Linthoudt D, Duvoisin B, Bovay P, Gerster JC. The cervical spine in calcium pyrophosphate dihydrate deposition disease: a prevalent casecontrol study. J Rheumatol 2004;31:545-9.

4. Kobayashi T, Miyakoshi N, Konno N, Abe E, Ishikawa Y, Shimada Y. Acute neck pain caused by arthritis of the lateral atlantoaxial joint. Spine J 2014;14:190913.

5. Constantin A, Marin F, Bon E, Fedele M, Lagarrigue B, Bouteiller G. Calcification of the transverse ligament of the atlas in chondrocalcinosis: computed tomography study. Ann Rheum Dis 1996;55:137-9.

6. Felson DT, Anderson JJ, Naimark A, Kannel W, 
Meenan RF. The prevalence of chondrocalcinosis in the elderly and its association with knee osteoarthritis: the Framingham Study. J Rheumatol 1989;16:1241-5.

7. Neame RL, Carr AJ, Muir K, Doherty M. UK community prevalence of knee chondrocalcinosis: evidence that correlation with osteoarthritis is through a shared association with osteophyte. Ann Rheum Dis 2003;62:513-8.

8. Inokuchi R, Ohshima K, Yamamoto M, Fukuda T, Nakamura K. Crowned dens syndrome. Spine J 2015;15:1499-500.

9. Kuriyama A. Crowned dens syndrome. CMAJ 2014;186:293.

10. Tajima K, Ueda T, Ueno K, Shimizu C, Yoshizawa J, Hori S. Crowned dens syndrome: reports of six cases and review of the literature. Acute Med Surg 2015;3:155-8.

11. Tamura T, Suzuki M, Hori S. Crowned dens syndrome. Intern Med 2015;54:545.

12. Yamada T, Saitoh T, Hozumi H, et al. Crowned dens syndrome. Acute Med Surg 2015;2:273.

13. Bundens WD Jr, Brighton CT, Weitzman G. Primary articular-cartilage calcification with arthritis (pseudogout syndrome). J Bone Joint Surg Am 1965;47:11122.

14. McCarty DJ, Kohn NN, Faires JS. The significance of calcium phosphate crystals in the synovial fluid of arthritic patients: the "pseudogout syndrome": I. clinical aspects. Ann Internal Med 1962;56(5_ Part_1):711-37. 